\title{
Computational split-field finite-difference time-domain evaluation of simplified tilt-angle models for parallel-aligned liquid-crystal devices
}

\author{
Andrés Márquez, a,b,* Jorge Francés, ${ }^{a, b}$ Francisco J. Martínez, ${ }^{a, b}$ Sergi Gallego, ${ }^{a, b}$ Mariela L. Álvarez, ${ }^{a, b}$ \\ Eva M. Calzado, ${ }^{a, b}$ Inmaculada Pascual, ${ }^{b, c}$ and Augusto Beléndez ${ }^{a, b}$ \\ aUniversidad de Alicante, Dept. de Física, Ing. de Sistemas y T. Señal, Alicante, Spain \\ I.U. Física Aplicada a las Ciencias y las Tecnologías Universidad de Alicante, Alicante, Spain \\ 'Universidad de Alicante, Farmacología y Anatomía, Dept. de Óptica, Alicante, Spain
}

\begin{abstract}
Simplified analytical models with predictive capability enable simpler and faster optimization of the performance in applications of complex photonic devices. We recently demonstrated the most simplified analytical model still showing predictive capability for parallel-aligned liquid crystal on silicon (PA-LCoS) devices, which provides the voltage-dependent retardance for a very wide range of incidence angles and any wavelength in the visible. We further show that the proposed model is not only phenomenological but also physically meaningful, since two of its parameters provide the correct values for important internal properties of these devices related to the birefringence, cell gap, and director profile. Therefore, the proposed model can be used as a means to inspect internal physical properties of the cell. As an innovation, we also show the applicability of the split-field finite-difference time-domain (SF-FDTD) technique for phase-shift and retardance evaluation of PA-LCoS devices under oblique incidence. As a simplified model for PA-LCoS devices, we also consider the exact description of homogeneous birefringent slabs. However, we show that, despite its higher degree of simplification, the proposed model is more robust, providing unambiguous and physically meaningful solutions when fitting its parameters. ๑ 2018 Society of Photo-Optical Instrumentation Engineers (SPIE) [DOI: 10.1117/1. OE.57.3.037110]
\end{abstract}

Keywords: liquid-crystal devices; parallel aligned; birefringence; spatial light modulators; displays; finite-difference time-domain; splitfield.

Paper 171566P received Oct. 2, 2017; accepted for publication Feb. 28, 2018; published online Mar. 24, 2018.

\section{Introduction}

Liquid crystal on silicon (LCoS) displays have become the most attractive microdisplays for all sorts of spatial light modulation applications, such as in optical imaging and metrology, ${ }^{1,2}$ optical storage, ${ }^{3,4}$ reconfigurable interconnects, ${ }^{5,6}$ or quantum optical computing, ${ }^{7}$ due to their very high spatial resolution and very high light efficiency. Among the different LCoS technologies, parallel-aligned LCoS (PA-LCoS $)^{8,9}$ is especially interesting. They offer unique capabilities as spatial light modulators (SLMs), since they enable phaseonly modulation without coupled amplitude modulation and with millions of addressable pixels.

PA-LCoS can be thought of as variable linear retarders whose linear retardance is changed by the applied voltage. Proper characterization of their linear retardance ensures optimal performance in applications. We proposed timeaveraged Stokes polarimetry ${ }^{10}$ as an advanced technique that enables robust linear retardance characterization in the presence of flicker, which is a typical degradation phenomenon found in most $\mathrm{LCoS}$ devices. ${ }^{11-13}$

In general, precise modeling of systems and devices requires the knowledge of a high number of different properties, which may not be provided by the manufacturer. Sometimes it is more important to follow a simpler yet

*Address all correspondence to: Andrés Márquez, E-mail: andres.marquez@ ua.es more intuitive approach where only the most significant relations and parameters are applied. Simplified models and/or reverse-engineering approaches, enabling analytical expressions, are then highly desirable, as it was the case with transmissive twisted-nematic liquid-crystal (LC) displays, ${ }^{14,15}$ which were the devices mostly used as SLMs until the appearance of modern PA-LCoS. We recently ${ }^{16}$ followed this approach to propose a simplified model for PA-LCoS microdisplays, especially suited for the large number of users of PA-LCoS devices, more interested in their wide range of applications than in a detailed modeling of the device itself.

For a complete description of operation of LC cells, a large number of parameters characterizing the LC material and the LC cell need to be known ${ }^{17}$ : ordinary and extraordinary refractive indices, cell gap, pre-tilt angle, index of refraction of the glass window, viscosity and elastic coefficients, electrode structure, among others. In contrast, our model represents, to our knowledge, the most simplified approach still showing predictive capability, as experimentally demonstrated ${ }^{16}$ It is based on only three parameters: two off-state (nonvoltage dependent) and one on-state (voltage dependent). At a lesser level of simplification, we have the exact expressions for a homogeneous birefringent slab, ${ }^{17,18}$ in the following the "reference" model. Both in the "proposed" and in the reference models, the nonhomogeneous tilt

0091-3286/2018/\$25.00 @ 2018 SPIE 
angle profile across the LC cell is neglected, among other phenomena such as Fresnel reflections, multiple interferences, and pre-tilt angle. In the proposed model, we further neglect double-refraction effects, and the magnitude of the birefringence is considered to be small with respect to the ordinary and extraordinary refractive indices. In spite of all these approximations, in our previous study, ${ }^{16}$ we obtained a good agreement between theoretical and experimental retardance values with errors smaller than $5 \%$. However, since in general construction parameters of the device and the LC material are not available, we cannot check in the laboratory experiment if the values obtained for the parameters in any of the two models are close to the correct ones.

We want to learn if the proposed model can be considered physically meaningful so that it can be used to probe into the internal properties of PA-LC devices. To meet this goal, we need to verify if the values obtained for the parameters are close to the correct ones. Recently, we presented some preliminary results ${ }^{19}$ indicating that the proposed model is actually able to probe into some of the internal properties of PA-LC devices. This analysis is done using as experimental retardance data the one provided by realistic simulations applying rigorous numerical electromagnetic approaches. First, we construct a virtual PA-LC cell. For the tilt angle profile, we consider a sine-like analytical approximation to the realistic nonhomogeneous voltage-dependent tilt angle profiles, ${ }^{17,20}$ which is easier to apply and the accuracy of which is good enough as long as the applied voltage is not very large.

Once we have generated the LC-director orientation profiles for a wide range of applied voltages, we can use different approaches to simulate rigorous light-matter interaction. A widely used rigorous electromagnetic approach is the finite-difference time-domain (FDTD) technique, ${ }^{21}$ applied in many areas of electromagnetics and which has also penetrated in recent years in the solution of problems in photonics. $^{22,23}$ To apply the periodic boundary conditions (PBC) in time-domain and oblique angle of incidence is challenging due to the phase difference (or time delay) between two periodic boundaries. In this work, the split-field FDTD (SF-FDTD) scheme is used instead of the standard FDTD analysis. SF-FDTD permits to analyze accurately periodic systems at optical wavelengths with oblique angle of incidence. This method removes the phase variation by means of a set of auxiliary transformed fields, avoiding the problems due to the standard FDTD scheme. Thus, many researchers have focused their efforts in this topic due to its potential for simulating photonic and diffractive optics elements. ${ }^{22-27}$ The SF-FDTD scheme economizes in memory resources, thus enabling the increase in spatial and time resolution of the discrete grid describing the device under test. It is the first time, to our knowledge, that is being applied to PA-LC devices under oblique incidence for phase-shift and retardance calculations. Phase calculations require an additional degree of accuracy when compared with more common intensity calculations.

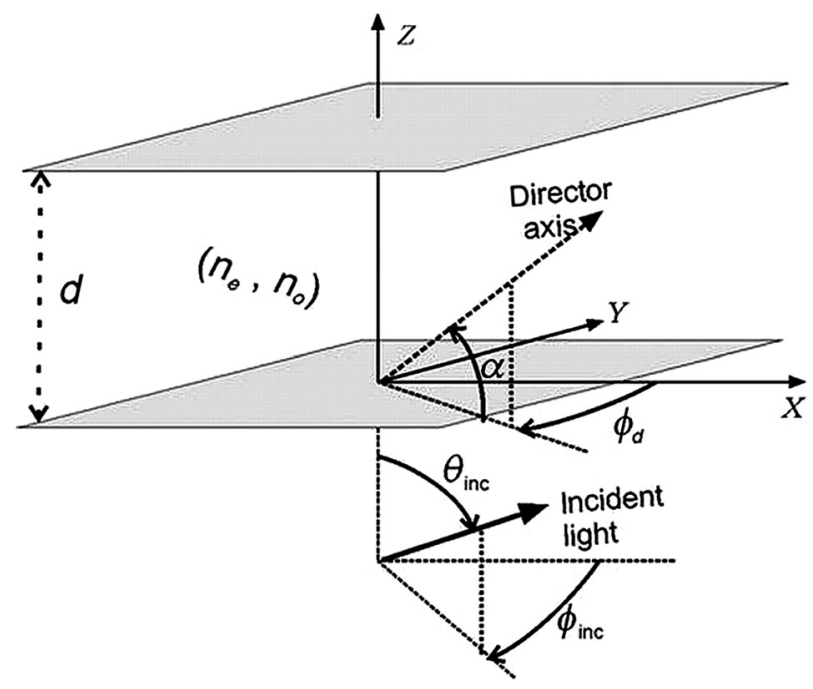

Fig. 1 Diagram for arbitrary light impinging in the reference model for the PA-LC cell.

In this paper, we present a deeper and more complete analysis than in our previous work, ${ }^{19}$ where we also compare the results provided by the reference model. This helps to delimitate the goodness and usefulness of the more simplified model proposed. We provide extensive details related to the design of the virtual PA-LC cell and the implementation of the SF-FDTD method. In Sec. 2, we introduce the proposed and the reference model, both of them homogeneous tilt angle models. In Sec. 3, we develop the details for the construction of the virtual PA-LC cell and the SF-FDTD computation. Results for the proposed and reference models are given in Sec. 4 and the main conclusions are found in Sec. 5.

\section{Homogeneous Tilt Angle Simplified Models}

\subsection{Reference Model}

Rigorous expressions for the retardance introduced by a homogeneous tilt-angle LC cell are provided in the literature $^{17,18}$ and are obtained by direct application of the Maxwell equations. Its diagram is shown in Fig. 1, where $\alpha$ and $\theta_{\text {inc }}$ are the tilt angle for the LC director and the angle of incidence for the light beam, respectively, $\phi_{d}$ and $\phi_{\text {inc }}$ are the corresponding azimuth angles, and $n_{o}$ and $n_{e}$ are the ordinary and the extraordinary refractive indices in the LC layer of thickness $d$, respectively. This rigorous approach includes the double refraction produced for a beam of light transmitted into the uniaxial medium and the phase retardation is given by

$\Gamma=\left(k_{e, z}-k_{o, z}\right) d$,

where $k_{e, z}$ and $k_{o, z}$ are the $z$-axis components of the wave vectors of the extraordinary and ordinary waves, respectively

$k_{e, z}=\frac{2 \pi}{\lambda} \frac{n_{e} n_{o}}{\varepsilon_{z z}}\left[\sqrt{\varepsilon_{z z}-\left(1-\frac{n_{e}^{2}-n_{o}^{2}}{n_{e}^{2}} \cos ^{2} \alpha \sin ^{2}\left(\phi_{d}-\phi_{\mathrm{inc}}\right)\right) \sin ^{2} \theta_{\mathrm{inc}}}-\frac{\varepsilon_{x z}}{\varepsilon_{z z}} \sin \theta_{\mathrm{inc}}\right]$, 


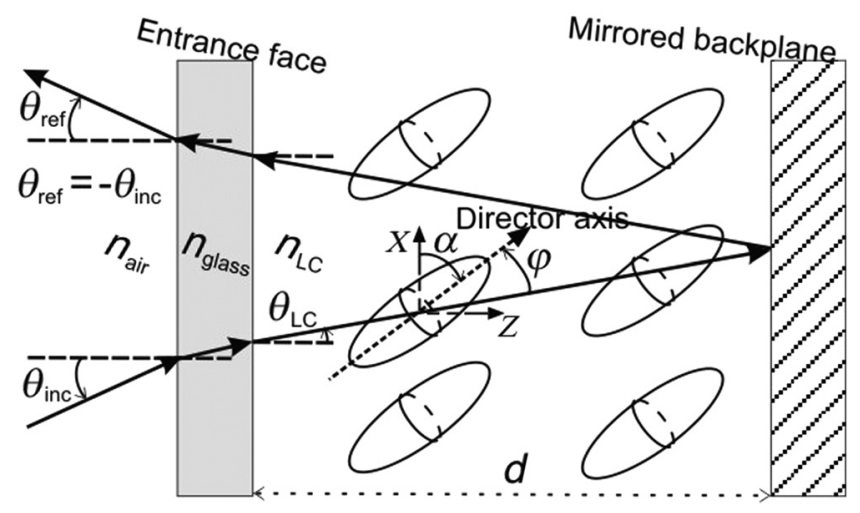

Fig. 2 Diagram for the PA-LC cell considered in the model proposed.

$k_{o, z}=\frac{2 \pi}{\lambda} \sqrt{n_{o}^{2}-\sin ^{2} \theta_{\text {inc }}}$,

and $\varepsilon_{x z}$ and $\varepsilon_{z z}$ are given by

$\varepsilon_{x z}=\left(n_{e}^{2}-n_{o}^{2}\right) \sin \alpha \cos \alpha \cos \left(\phi_{d}-\phi_{\text {inc }}\right)$,

$\varepsilon_{z z}=n_{o}^{2}+\left(n_{e}^{2}-n_{o}^{2}\right) \sin ^{2} \alpha$.

The basic working configuration in most applications considers the LC-director axis along or perpendicular to the incidence plane. ${ }^{28,29}$ We will consider the specific situation where $\phi_{\text {inc }}=\phi_{d}=0 \mathrm{deg}$, i.e., LC director along $X Z$ which is the incidence plane.

\subsection{Proposed Model}

Detailed development of the proposed model can be found in our initial paper, ${ }^{16}$ where its validity was demonstrated against experimental measurements from a commercial reflective PA-LCoS device. When compared with the reference model, in our proposed model, we further neglect double-refraction effects, and the magnitude of the birefringence is considered to be small with respect to the ordinary and extraordinary refractive indices. In Fig. 2, we show the general diagram for a reflective cell with a cell gap $d$. Incidence plane and LC director are along the $X Z$ plane as in the case of the reference model in Sec. 2.1. LC molecules have their director axis (optical axis) aligned at an angle $\varphi$ with respect to the traversing light beam direction. $\theta_{\mathrm{LC}}$ is the refraction angle in the LC medium. The director axis tilts an angle $\alpha$ with respect to the entrance face as a function of the applied voltage $\mathrm{V}$. This is the only voltage-dependent magnitude, i.e., $\alpha(V)$. At the backplane, the light beam is reflected and a second passage is produced across the LC layer whose effect is equivalent to a forward propagation at an angle $-\theta_{\text {inc }}$. In the model, we define two off-state parameters, combination of the LC indices ordinary and extraordinary, $n_{o}$ and $n_{e}$, together with the cell gap $d$. These parameters are $\mathrm{OPL}=d n_{o}$ and $\mathrm{OPD}=d \Delta n$, which correspond to the magnitudes of the optical path length for the ordinary component and the optical path difference between extraordinary and ordinary components, respectively.

As the starting point for the derivation of the simplified model, we consider that for a uniaxially anisotropic material, as it is the case in most of LCs, the effective extraordinary index $n_{\text {eff }}$ depends on the angle $\varphi$ between director axis and light beam as follows:

$$
\frac{1}{n_{\mathrm{eff}}^{2}(\varphi)}=\frac{\sin ^{2} \varphi}{n_{e}^{2}}+\frac{\cos ^{2} \varphi}{n_{o}^{2}} .
$$

The retardance $\Gamma$ between the extraordinary and ordinary components is given by

$\Gamma=\frac{2 \pi}{\lambda} \frac{d}{\cos \theta_{\mathrm{LC}}}\left[n_{\mathrm{eff}}(\varphi)-n_{o}\right]$

where $\lambda$ is the wavelength of the light beam and $d / \cos \theta_{\mathrm{LC}}$ is the length of the trajectory across the LC layer. We show ${ }^{16}$ that application of the approximation $n_{o} \gg \Delta n$ and the following substitutions $\mathrm{OPL}=d n_{o}$ and $\mathrm{OPD}=d \Delta n$ lead to the following analytical expression for the retardance:

$\Gamma=\frac{2 \pi}{\lambda} \frac{\mathrm{OPL}}{\cos \theta_{L C}}\left[\frac{1+(\mathrm{OPD} / \mathrm{OPL})}{1+(\mathrm{OPD} / \mathrm{OPL}) \cos ^{2} \varphi}-1\right]$.

According to Fig. 2, angle $\varphi$ is given by

$\varphi\left(\theta_{\mathrm{inc}}, V\right)=\frac{\pi}{2}+\alpha(V) \mp \theta_{\mathrm{LC}}\left(\theta_{\mathrm{inc}}\right)$,

where the $\mp$ sign applies for the forward (backward) passage. The total retardance in the PA-LCoS is given by the addition of the forward and backward retardances. In the case of normal incidence and LC-director axis parallel to the entrance face, then Eq. (8) simplifies into the well-known expression $\Gamma=2 \pi d \Delta n / \lambda$. Our model produces a much simpler expression and reduces the number of parameters when compared with the exact expressions for a homogeneous uniaxial anisotropic plate, i.e., the reference model.

The voltage-dependent tilt angle $\alpha(V)$ does not depend on the wavelength. However, OPD and OPL values depend on the illumination wavelength considered. Once they are fitted at the specific wavelengths used in the experiments for calibration, then we can interpolate them at other wavelengths. The extended Cauchy relation provides a good fit for OPD ${ }^{16}$ whereas for OPL values we have to use the basic linear interpolation. As a whole, we are able to obtain the retardance not only for a wide range of incidences (to $45 \mathrm{deg}$ ) but also across the wavelengths in the visible region of the spectrum. 


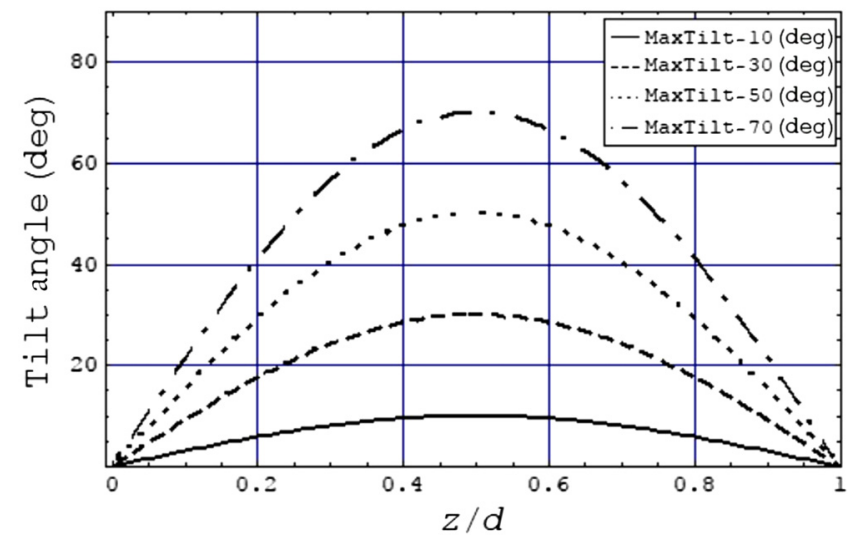

Fig. 3 LC-director sine-like tilt angle profiles across the thickness of the cell and for various $\alpha_{\max }$ values.

\section{SF-FDTD Computational Experiment}

\subsection{Virtual PA-LC Cell with Sine-Like Tilt Angle Profile}

To evaluate the physical significance of the three parameters in the model, we consider the nematic LC E7 at room temperature $\left(20^{\circ} \mathrm{C}\right)$ and a cell gap of $2 \mu \mathrm{m}$. The LC mixture $\mathrm{E} 7$ is one of the classical compounds found in the literature dealing with LC devices. ${ }^{30}$ We apply a sine-like tilt angle profile, which is a valid approximation as long as the applied voltages are small enough not to produce a saturation in the tilt angle. In many situations, availability of analytical expressions is a very interesting situation since parameters are then easier to analyze to obtain the necessary in-depth physical insight. In the case of tilt angle profiles, there is also an interest in obtaining analytic expressions for the tilt angle profiles as done by Abdulhalim and Menashe. ${ }^{20}$ In the sine profile, the tilt angle $\alpha$ across the cell thickness $z$ varies as

$\alpha=\alpha_{\max } \sin (\pi z / d)$,

where $d$ is the cell gap and $\alpha_{\max }$ is the maximum tilt angle, which occurs in the midlayer of the cell. In Fig. 3, we show the sine-like tilt profile for various $\alpha_{\max }$ values. We display the normalized thickness parameter $z^{\prime}=z / d$ in the $X$-axis. We consider $\alpha_{\max }$ values in the interval from 0 deg to $70 \mathrm{deg}$ since, as we will see in Sec. 4.2, when fitting the on-state parameter $\alpha(V)$ we obtain that its values are within the range found already in commercial devices. ${ }^{16}$

As refractive indices, we use the tabulated values provided in the paper by $\mathrm{Li}$ et al. $^{30}$ and interpolate them using the Cauchy relation to produce additional values at other wavelengths. In Table 1, we show the values for the ordinary and the extraordinary refractive indices at wavelengths 633, 532, and $473 \mathrm{~nm}$, which are the ones selected to compare with the results provided by the simplified model. Indeed, the retardance is calculated for a total of 18 different equidistant wavelengths running from 473 to $634 \mathrm{~nm}$ across the whole visible spectrum.

\subsection{SF-FDTD Computation}

Once we generate the LC-director profiles, then we apply the SF-FDTD method to simulate the propagation of the incident
Table 1 Values for the refractive indices for the three wavelengths specifically analyzed in the paper.

\begin{tabular}{lcc}
$\lambda(\mathrm{nm})$ & $n_{e}$ & $n_{o}$ \\
\hline 633 & 1.7371 & 1.5189 \\
532 & 1.7646 & 1.5289 \\
473 & 1.7935 & 1.5384 \\
\hline
\end{tabular}

electromagnetic field across the PA-LC cell. In Fig. 4, we show a general diagram of the structure for the SF-FDTD simulation space. We consider the LC cell composed of the LC layer with a perfectly conducting mirror at the rear surface, respectively, the anisotropic medium and the mirror perfectly electric conductive in Fig. 4. The glass window at the entrance is not considered. This has only a residual impact in the degree of realism of the simulation and has the benefit of reducing significantly the number of points, thus the memory resources needed, sampling the cell structure: the LC layer is $2-\mu \mathrm{m}$ thick, whereas the glass window is typically a few millimeters thick, thus needing many more points in the sampling grid. To the entrance interface air-LC layer, we add an antireflection (AR) thin film structure for the visible spectrum, ${ }^{31,32}$ mimicking the usual AR coating found in PA-LCoS devices. We have also produced results with no AR coating that we do not show in this paper; the effect of the multiple interferences was very noticeable and was not representative of the experimental results obtained with usual LCoS devices, ${ }^{16}$ even though in some cases it might be important or even interesting. ${ }_{33,34}$

As in any FDTD simulation, we need to introduce the perfectly matched layers, ${ }^{21}$ in this case for anisotropic materials, at the edges of the modeling window to enforce absorbing boundary conditions to avoid nonphysical reflection of the

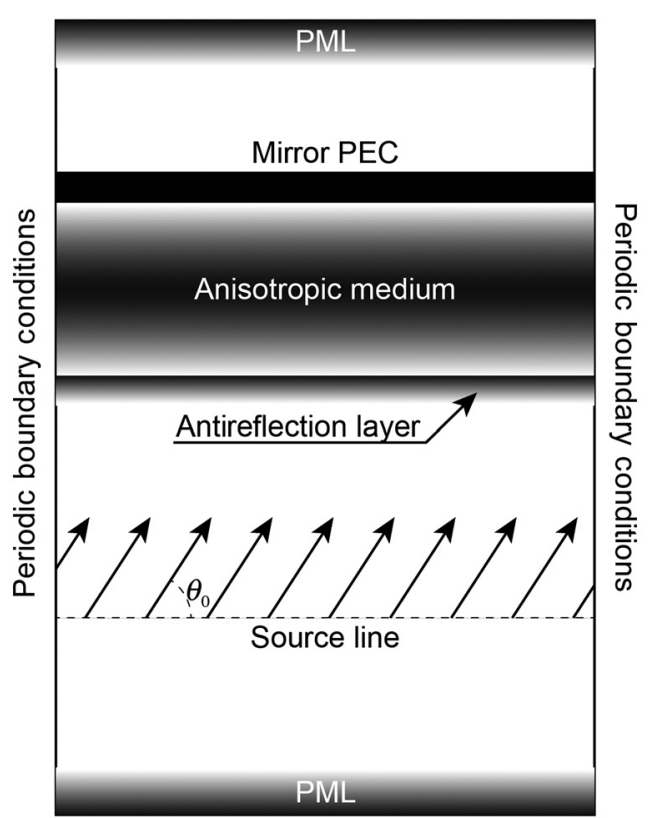

Fig. 4 Diagram of the structure for the SF-FDTD simulation grid. 


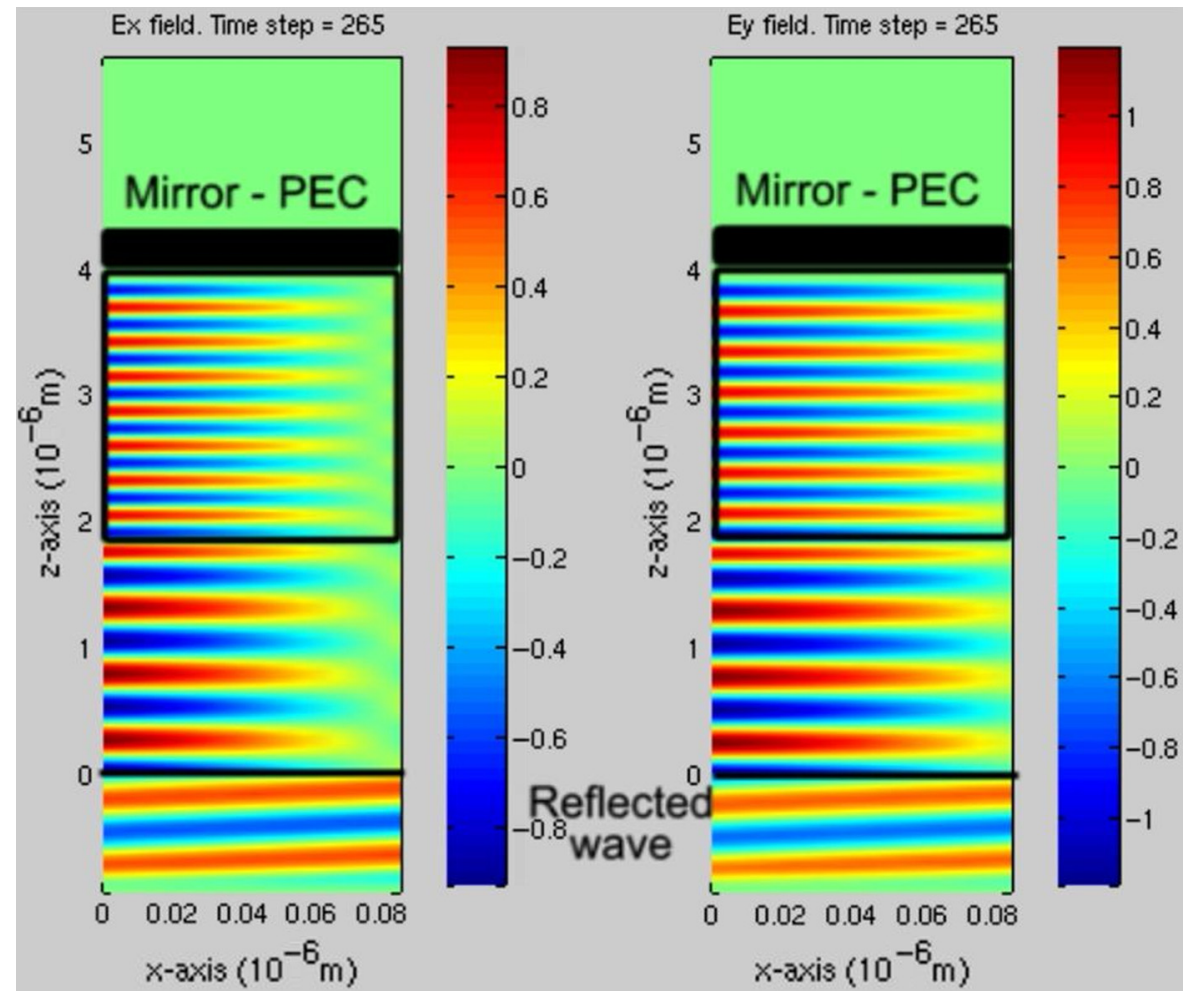

Fig. 5 Time evolution of the TE and TM electric field components across the simulation grid. The X-component is along the incidence plane (TM) and parallel to the LC director (Video 1, MPEG, $2.5 \mathrm{MB}$ [URL: https://doi.org/10.1117/1.OE.57.3.037110.1]).

considered optical field at these edges. In the SF implementation for a periodic structure, we consider one single period and we apply the appropriate PBC at its sides. In our case, where the structure is not periodic, the benefit of applying the SF technique is that we only need to consider a small transverse portion of the cell to produce the same results of an infinite transverse extent, thus the size of the simulation is highly decreased when compared with the conventional FDTD methodology. We also need to apply the Courant condition $^{21}$ to ensure stability and convergence of the method. In Fig. 4, we also show the location of the line source in front of the anisotropic medium and producing oblique incidence.

The goal is to reproduce computationally the measurement and data reduction methodology followed in the lab experiment. ${ }^{10}$ In the lab, this is accomplished by illuminating the PA-LC structure with a light beam linearly polarized at $45 \mathrm{deg}$ with respect to the director axis of the LC layer at the entrance face. For the reflected light beam, we measure its Stokes parameters, and from the ratio between the third and fourth Stokes vector components, the retardance $\Gamma$ value is calculated as follows: $\Gamma=\tan ^{-1}\left(S_{3} / S_{2}\right)$.

In the simulation, the SF-FDTD provides the values for the amplitude of the electric field at the output of the reflective PA-LC cell. This enables to calculate the phase shift between the electric field component polarized along the LC-director axis and the one orthogonally polarized. This phase shift is the retardance $\Gamma$ of the PA-LC cell. In the simulation, we monitor this difference and make sure that transient effects disappear and a steady-state regime is obtained. We stop the simulation after 5000 temporal steps, equivalent to about 0.03 ps. This duration varies slightly depending on the obliquity of the angle of incidence since the Courant condition depends on the angle of incidence. We consider angles of incidence at $0 \mathrm{deg}$ (normal incidence), $3 \mathrm{deg}$, $25 \mathrm{deg}, 35 \mathrm{deg}$, and $45 \mathrm{deg}$, which cover a wide range of working conditions found in applications. ${ }^{28,29}$ The excitation of the LC layer at each point in the sampling grid by the incident electric field is given by the dielectric permittivity tensor $\overline{\bar{\varepsilon}}$

$\overline{\bar{\varepsilon}}=\left(\begin{array}{ccc}\varepsilon_{\|} \cos ^{2}\left(\alpha^{\prime}\right)+\varepsilon_{\perp} \sin ^{2}\left(\alpha^{\prime}\right) & 0 & \varepsilon_{\|} \cos \left(\alpha^{\prime}\right) \sin \left(\alpha^{\prime}\right)-\varepsilon_{\perp} \cos \left(\alpha^{\prime}\right) \sin \left(\alpha^{\prime}\right) \\ 0 & \varepsilon_{\perp} & 0 \\ \varepsilon_{\|} \cos \left(\alpha^{\prime}\right) \sin \left(\alpha^{\prime}\right)-\varepsilon_{\perp} \cos \left(\alpha^{\prime}\right) \sin \left(\alpha^{\prime}\right) & 0 & \varepsilon_{\|} \sin ^{2}\left(\alpha^{\prime}\right)+\varepsilon_{\perp} \cos ^{2}\left(\alpha^{\prime}\right)\end{array}\right)$,

resulting from the application of the corresponding Euler angles for the PA-LC cell geometry: $\alpha=0 \mathrm{deg}, \beta=90 \mathrm{deg}-\alpha^{\prime}$, and $\gamma=0 \mathrm{deg}$, where $\alpha^{\prime}$ corresponds to the tilt angle in the cell. In Fig. 5, we show the time evolution of electric field from the moment the line source starts emitting the wavefront until the reflected electric field reaches the steady state. The left part corresponds to the transversal magnetic (TM) component and lies parallel to the LC director. In the time evolution, we see that the TM-reflected wave has a time delay with respect to the TE-reflected wave. The LC E7 has positive dielectric 


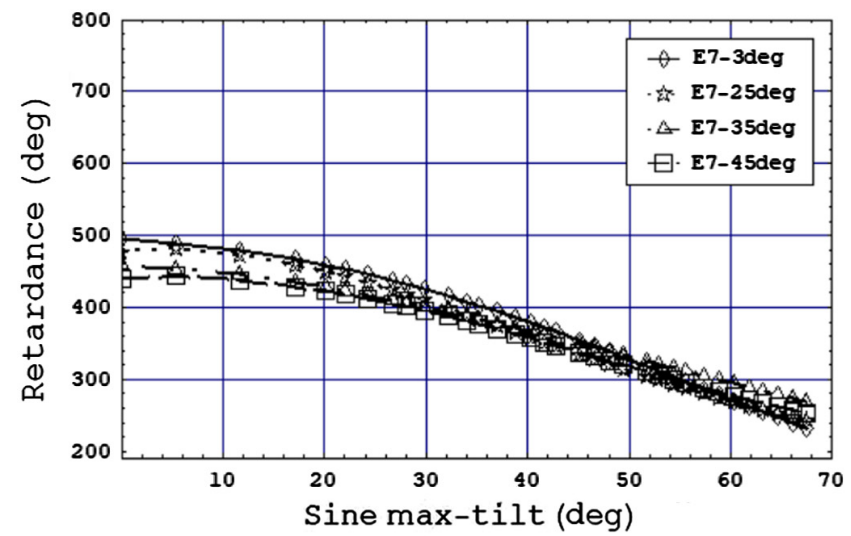

(a)

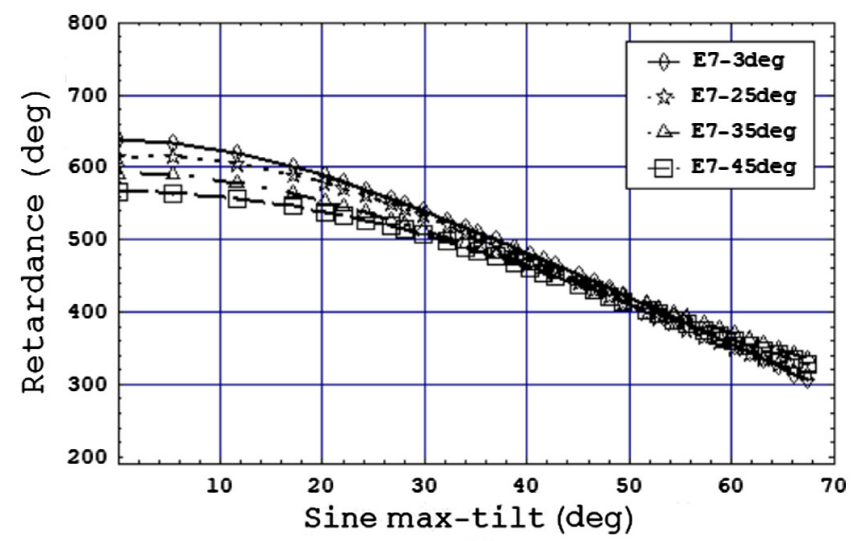

(b)

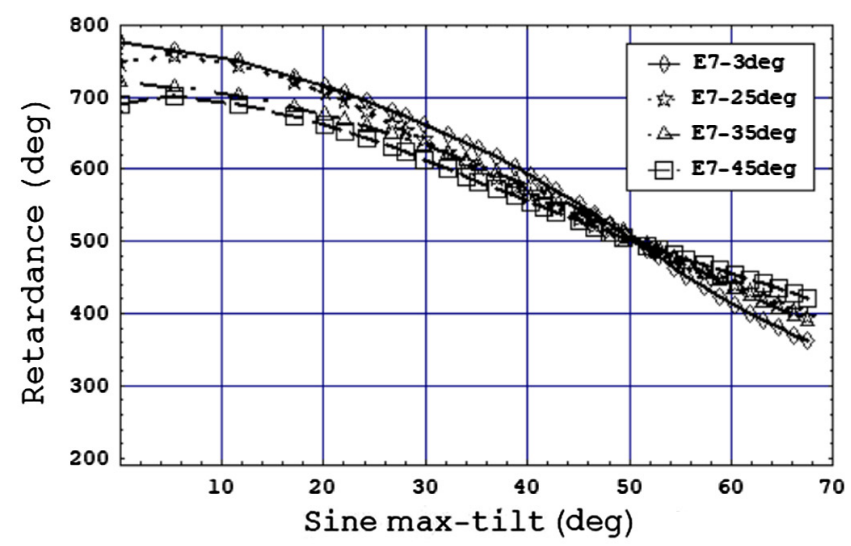

(c)

Fig. 6 Retardance SF-FDTD simulated measurements for the various incidence angles (in the legend) and for the wavelengths: (a) $633 \mathrm{~nm}$, (b) $532 \mathrm{~nm}$, and (c) $473 \mathrm{~nm}$.

anisotropy, i.e., $n_{e}>n_{o}$ and thus the extraordinary component propagates slower. This is the typical situation with nematic LCs.

We note that to be sure that our SF-FDTD implementation is correct and accurate enough, first thing we have done is to reproduce the experimental retardance measurements presented in our previous work, ${ }^{16}$ assuming a sine-like LC-director profile across the cell. Thus, we have checked that our SF-FDTD implementation provides correct retardance values.

In Figs. 6(a), 6(b), and 6(c), we show the retardances as a function of $\alpha_{\max }$ calculated using the SF-FDTD approach
Table 2 Values for the retardance in the off-state.

\begin{tabular}{lccccc}
\hline & \multicolumn{5}{c}{ Incidence angle (deg) } \\
\cline { 2 - 6 }$\lambda(\mathrm{nm})$ & 0 & 3 & 25 & 35 & 45 \\
\hline 633 & 496.4 & 496.1 & 476.8 & 459.6 & 439.3 \\
532 & 638.0 & 637.6 & 613.1 & 591.4 & 565.7 \\
473 & 776.6 & 776.2 & 746.7 & 720.6 & 689.7 \\
\hline
\end{tabular}

for the virtual PA-LC explained in Sec. 3.1 and for the three wavelengths 633,532 , and $473 \mathrm{~nm}$, respectively. We decide to represent angles at $3 \mathrm{deg}, 25 \mathrm{deg}, 35 \mathrm{deg}$, and $45 \mathrm{deg}$ (see legend) since results at 0 deg and 3 deg are almost equal. We clearly appreciate the nonlinear monotonous decrease of retardance with $\alpha_{\max }$. We also verify that the retardance dynamic range becomes shorter for larger angles of incidence, which was also found in the experimental measurements in previous papers. ${ }^{28,29}$

We also produce by simulation the values for the off-state, i.e., when no voltage is applied to the device. To this goal, we directly consider the results produced by the homogeneous uniaxial anisotropic slab expressions, given in Sec. 2.1; in the off-state, the LC device has a uniform LC-director distribution and the exact expression can be used with a higher accuracy. In Table 2, we show the retardance for the five angles of incidence and for the three wavelengths for the off-state.

\section{Numerical Results}

\subsection{Off-State Analysis}

In this section, we fit the off-state parameters. Afterward, in Sec. 4.2, we fit the tilt angle, which depends on the applied voltage. We will consider both the reference and the proposed models. As experimental values for the off-state calibration, we consider the simulated retardances shown in Table 2 for the angles of incidence of $3 \mathrm{deg}$ and $35 \mathrm{deg}$ and for the wavelengths 633, 532, and 473. Both for the reference and the proposed models, the off-state parameters are wavelength dependent but do not depend on the angle of incidence.

In Table 3, we show various solution sets obtained from the calibration with the reference model. The figure of merit $\chi^{2}$ to be minimized combines two squared differences: on one hand, between theoretical and simulated retardance values normalized by the simulated value, and on the other hand, between the theoretical and simulated ratios of the retardance values for the pair of incidence angles considered ( $3 \mathrm{deg}$ and $35 \mathrm{deg}$ ) normalized by the simulated ratio. These two normalized squared differences are added up for the three wavelengths. This minimized value for $\chi^{2}$ is given for each set of fitted parameters in Table 3 (second row). Then, in the third row, we show the mean square error (MSE) difference, which serves to validate the goodness of the agreement between theory and experiment for the onstate fitting of the tilt angle $\alpha(V)$ parameter. The figure of merit MSE is given by the square difference at each voltage between the theoretical and simulated retardance values normalized by the simulated value, added up for the whole range 
Table 3 Some of the solution sets using the reference model. Fitting off-state retardance values for 633,532 , and $473 \mathrm{~nm}$. Starting values in parentheses (bold-face when different from the true values). Resulting values italicized when different from the true ones. Figures of merit for the off-state $\chi^{2}$ and for the on-state MSE fittings in second and third rows.

\begin{tabular}{|c|c|c|c|c|c|}
\hline & Set\#1 & Set\#2 & Set\#3 & Set\#4 & Set\#5 \\
\hline$\chi^{2}$ off-state & $1.98 \times 10^{-28}$ & $1.20 \times 10^{-29}$ & $5.92 \times 10^{-29}$ & $3.18 \times 10^{-29}$ & $6.00 \times 10^{-28}$ \\
\hline MSE on-state & 0.00047 & 0.00048 & 0.00047 & 0.00047 & 0.00046 \\
\hline$d(\mu \mathrm{m})$ & $2.00(2.00)$ & $1.85(3.00)$ & $2.25(2.00)$ & $2.16(2.00)$ & $3.79(4.00)$ \\
\hline$n_{e}(633 \mathrm{~nm})$ & $1.7371(1.7371)$ & $1.7554(1.7371)$ & $1.7133(1.7071)$ & $1.7213(1.7071)$ & $1.6341(1.7071)$ \\
\hline$n_{o}(633 \mathrm{~nm})$ & 1.5189 (1.5189) & 1.5189 (1.5189) & 1.5189 (1.5189) & 1.5189 (1.4189) & 1.5189 (1.4189) \\
\hline$n_{e}(532 \mathrm{~nm})$ & $1.7646(1.7646)$ & $1.7844(1.7646)$ & $1.7389(1.7146)$ & $1.7476(\mathbf{1 . 7 1 4 6 )}$ & $1.6533(1.7146)$ \\
\hline$n_{o}(532 \mathrm{~nm})$ & 1.5289 (1.5289) & 1.5289 (1.5289) & 1.5289 (1.5289) & 1.5289 (1.4289) & 1.5289 (1.4289) \\
\hline$n_{e}(473 \mathrm{~nm})$ & 1.7935 (1.7935) & $1.8149(1.7935)$ & $1.7657(1.7235)$ & $1.7751(\mathbf{1 . 7 2 3 5 )}$ & $1.6730(1.7235)$ \\
\hline$n_{o}(473 \mathrm{~nm})$ & $1.5384(1.5384)$ & $1.5384(1.5384)$ & $1.5384(1.5384)$ & $1.5384(1.4384)$ & $1.5384(1.4384)$ \\
\hline
\end{tabular}

of voltages and for both incidences at $3 \mathrm{deg}$ and $35 \mathrm{deg}$, and then divided by the total number of samples to produce a mean value. Specific on-state results will be later shown in Sec. 4.2 .

We note that the theoretical expressions are nonlinear, and to start the iterative optimization process we have to assign initial values to the parameters. Each of the five different solution sets corresponds to a different set of starting iteration values. These starting values are indicated in parentheses. For the set\#1, the starting values are the true values given in Table 1 for LC E7 employed in this work. We see that the results produced by the optimization procedure are equal to the true values. For the set\#2, we change the starting value for the cell gap to $3 \mu \mathrm{m}$ (in bold face) and we see that the results obtained for the parameters deviate from the true values in the cell gap and in the extraordinary indices for the three wavelengths, which we show as italicized. In sets \#3, $\# 4$, and $\# 5$, we have starting values different from the true values for some of the parameters, which we show in bold face. We see that in general the resultant fitted values do not converge back into the true values. We also note that the figures of merit $\chi^{2}$ and MSE are very close for all the solution sets. This means that we do not have a means to distinguish which solution set is closer to the actual true values. In Table 3, we have only played with starting values not very far from the true values, but the same ambiguity in the solution is reached even when the starting values are very far from the true values.

Now we fit the off-state parameters for the proposed model, OPD and OPL, using the off-state retardance values in Table 2, as we have done for the reference model in

Table 4 Values for OPD and OPL calculated from the cell gap and the indices of refraction in Table 1.

\begin{tabular}{lccc}
$\lambda(\mathrm{nm})$ & 633 & 532 & 473 \\
\hline OPD $(\mu \mathrm{m})$ & 0.4364 & 0.4714 & 0.5102 \\
OPL $(\mu \mathrm{m})$ & 3.0378 & 3.0578 & 3.0768 \\
\hline
\end{tabular}

Table 3. To serve as a reference, in Table 4, we give the true values for OPD and OPL calculated according to the refractive indices and cell gap value from Table 1 . The fitted values obtained for OPD and OPL are given in Table 5, together with the values for the figures of merit $\chi^{2}$ and MSE, and for different values of $n_{\mathrm{LC}}$ (first row). We note that, in contrast with the reference model, our model provides the same resulting OPD and OPL values for a very wide range of parameters starting values, thus not showing multiple, i.e., ambiguous, solutions. This is something that we already showed against experimental results ${ }^{16}$ and now in this work we have consistently verified this result with the simulated experiments.

Something that we have found in the computational experiments is that the resulting OPD and OPL values depend on the index of refraction considered for the LC, parameter $n_{\mathrm{LC}}$. That is why we show in each of the columns in Table 5 the different solution sets obtained considering different values for $n_{\mathrm{LC}}$. We note that simple application of the Snell law to a plain parallel isotropic slab shows that the index of refraction for the glass window, $n_{\text {glass }}$ in Fig. 2, has no effect on the refraction angle $\theta_{\mathrm{LC}}$ inside of the LC layer. Only the value $n_{\text {air }}=1$ and the one assigned for $n_{\mathrm{LC}}$ determine $\theta_{\mathrm{LC}}$ through the Snell law expression, $\theta_{\mathrm{LC}}=\arcsin \left[\sin \left(\theta_{\text {inc }}\right) / n_{\mathrm{LC}}\right]$, where $\theta_{\text {inc }}$ is the angle of incidence in air (Fig. 2). In Table 5, we have chosen $n_{\mathrm{LC}}$ values ranging from 1.5 to 1.7 which fall within the typical range of values for LCs. Specifically, if we take into account the refractive indices for E7 in Table 1, the average refractive index, calculated as $\left(n_{e}+2 n_{o}\right) / 3$, is $1.59,1.61$, and 1.62, respectively, for the wavelengths 633,532 , and $473 \mathrm{~nm}$, and the global average of the three values is 1.61 .

The best MSE in Table 5 is obtained for $n_{\mathrm{LC}}=1.50$, where we obtain clearly a worse $\chi^{2}$. This seems contradictory but looking at the MSE values, we see that they are very close to each other through the whole range of $n_{\mathrm{LC}}$ simulated. If we take a look at the OPD values, we see that they are similar to the four decimal number with respect to the true values (Table 4), except for $n_{\mathrm{LC}}=1.50$ where it is similar to the third decimal number, which is still a very good agreement. Values for OPL show a larger range of 
Table 5 OPD and OPL values obtained from the fitting procedure and for different values for $n_{\mathrm{LC}}$. Figures of merit for the off-state $\chi^{2}$ and for the on-state MSE comparison between theoretical and experimental results in second and third rows.

\begin{tabular}{|c|c|c|c|c|c|c|c|}
\hline$n_{\mathrm{LC}}$ & 1.50 & 1.59 & 1.60 & 1.61 & 1.62 & 1.65 & 1.70 \\
\hline$\chi^{2}$ off-state & $4.8 \times 10^{-5}$ & $1.8 \times 10^{-24}$ & $7.1 \times 10^{-28}$ & $2.2 \times 10^{-18}$ & $2.8 \times 10^{-20}$ & $3.7 \times 10^{-26}$ & $2.5 \times 10^{-20}$ \\
\hline \multirow[t]{2}{*}{ MSE on-state } & 0.00050 & 0.00059 & 0.00060 & 0.00060 & 0.00060 & 0.00061 & 0.00063 \\
\hline & $(\mu \mathrm{m})$ & $(\mu \mathrm{m})$ & $(\mu \mathrm{m})$ & $(\mu \mathrm{m})$ & $(\mu \mathrm{m})$ & $(\mu \mathrm{m})$ & $(\mu \mathrm{m})$ \\
\hline OPD $(633 \mathrm{~nm})$ & 0.4369 & 0.4364 & 0.4364 & 0.4364 & 0.4364 & 0.4364 & 0.4364 \\
\hline OPL (633 nm) & $24.02 \times 10^{6}$ & 7.8298 & 6.8456 & 6.0775 & 5.4614 & 4.1772 & 2.9829 \\
\hline OPD (532 nm) & 0.4722 & 0.4714 & 0.4714 & 0.4714 & 0.4714 & 0.4714 & 0.4714 \\
\hline OPL (532 nm) & $39.89 \times 10^{6}$ & 9.9576 & 8.5336 & 7.4609 & 6.6237 & 4.9421 & 3.4506 \\
\hline OPD $(473 \mathrm{~nm})$ & 0.5113 & 0.5102 & 0.5102 & 0.5102 & 0.5102 & 0.5102 & 0.5102 \\
\hline OPL (473 nm) & $49.53 \times 10^{6}$ & 12.9004 & 10.7767 & 9.2462 & 8.0910 & 5.8679 & 3.9977 \\
\hline
\end{tabular}

variation and we also observe that they do not coincide with the true values. We conclude that the OPD parameter is physically meaningful and it does not depend on the value considered for $n_{\mathrm{LC}}$, thus, it is very robust. If we know the value for the cell gap by some other means, then the proposed model is able to provide reliable birefringence values as a function of wavelength.

\subsection{On-State Analysis}

Once we have the off-state parameters both for the reference and for the proposed model, we fix these values in the theoretical expressions as shown in Secs. 2.1 and 2.2 for each of the two models. In the case of the reference model, we consider the solution set\#1 in Table 3 (equal to the true values), and for the proposed model, we consider the solution in Table 5 obtained when $n_{\mathrm{LC}}=1.60$, where $\chi^{2}$ shows the lowest value.

Now, in a second step, we use the on-state SF-FDTD retardance values for incidence angles at $3 \mathrm{deg}$ and $35 \mathrm{deg}$ (shown in Fig. 6) as the experimental values for the fitting procedure with the theoretical expressions. From this fit, we obtain the tilt angle as a function of voltage $\alpha(V)$. In

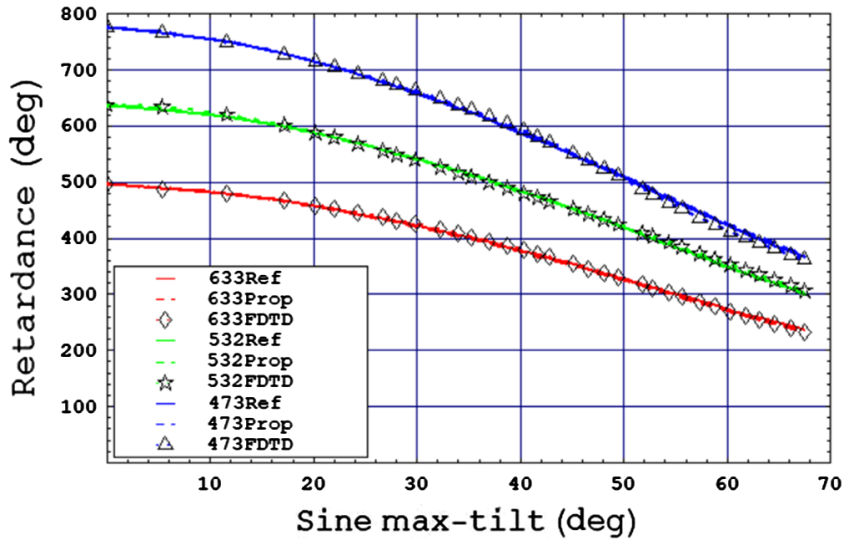

(a) this work, the role played by the voltage is actually expressed as a function of the maximum tilt angle $\alpha_{\max }$. Then the fitting procedure provides the relation $\alpha\left(\alpha_{\max }\right)$. The same figure of merit function $\chi^{2}$ previously explained in Sec. 4.1 is now used for the on-state fitting procedure, and the optimization is run independently for each of the $\alpha_{\max }$ values. In Figs. 7(a) and 7(b), we show the retardance versus voltage plots for the theoretical fitting using the reference and the proposed models (continuous and dashed lines) and the SF-FDTD simulated experimental data (dots) for the incidence angles at $3 \mathrm{deg}$ and $35 \mathrm{deg}$, and for the three wavelengths. We note that for both models theoretical and FDTD results agree very well with each other at both incidences and for the three wavelengths.

In Fig. 8, we show the fitted tilt angle for both the reference and the proposed models versus $\alpha_{\max }$. We also show the "average tilt" corresponding to the mean tilt value across the cell gap for the sine-like tilt profiles, as shown in Fig. 3. The "average" tilt follows a linear line, whereas the "proposed" tilt runs in parallel most of the range with a value about 4 deg larger, and the "reference" lies in between. We might think of the proposed and reference tilt angle as a corrected average tilt able to provide correct values for

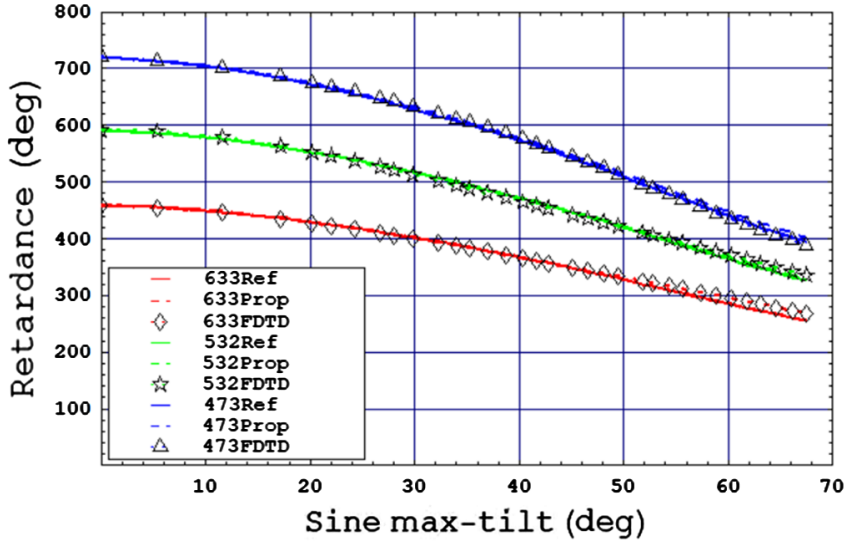

(b)

Fig. 7 SF-FDTD-experiment and theoretical fitting with the reference and the proposed models for the wavelengths 633,532 , and $473 \mathrm{~nm}$ and for incidence at (a) 3 deg and (b) 35 deg. 


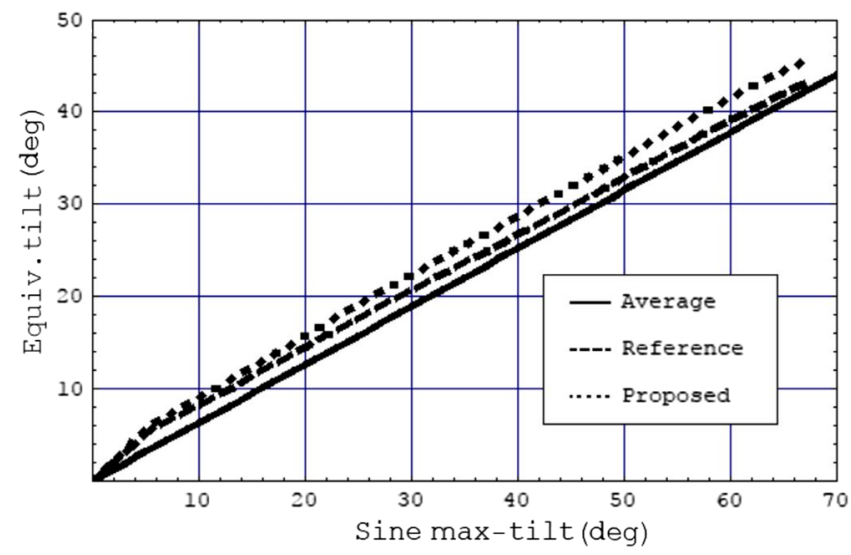

Fig. 8 Fitted equivalent tilt angle as a function of $\alpha_{\max }$ for the sine profile.

the retardance of the nonhomogeneous profiles. We note that we have limited our attention to a maximum $\alpha_{\max }$ of about 70 deg; within this range, the fitted equivalent tilt angle runs from 0 deg to about $45 \mathrm{deg}$, which is within the range we found for commercial PA-LCoS microdisplays. ${ }^{16}$

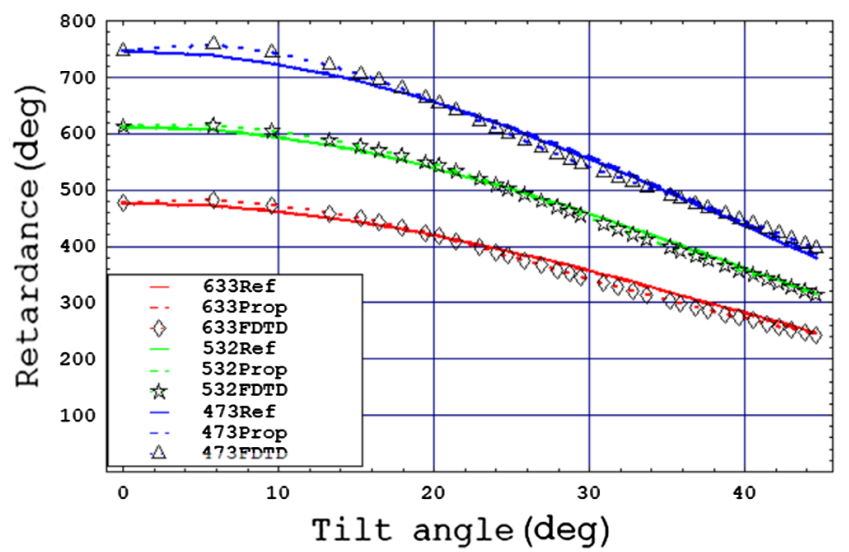

(a)
Now, using the fitted tilt angle in Fig. 8, we test the predictive capability for both the reference and the proposed models at two other angles of incidence not used in the calibration. In Figs. 9(a) and 9(b), respectively, for incidence at $25 \mathrm{deg}$ and $45 \mathrm{deg}$, we show both the theoretical values calculated with the models (continuous and dash line) and the SF-FDTD values (dots). Applying the curves in Fig. 8, now the retardance is plotted as a function of the corresponding fitted tilt angle. As commented in the previous paper, ${ }^{16}$ this helps to make more explicit that the actual dependence of retardance is with the tilt angle. We note the good agreement between model and SF-FDTD experiment.

To provide a more quantitative evaluation of the agreement between the theoretical predictions and the simulated SF-FDTD experimental data points, we calculate the normalized retardance difference (theoretical minus SF-FDTD experimental retardance normalized by the theoretical values). In Figs. 10(a) and 10(b), respectively, for angles of incidence $25 \mathrm{deg}$ and $45 \mathrm{deg}$, we show these values for the reference model, and in Figs. 11(a) and 11(b) equivalently for the proposed model. Both simplified models, the reference and the proposed, predict the retardance with relative uncertainties that in most of the tilt angle range is $<5 \%$.

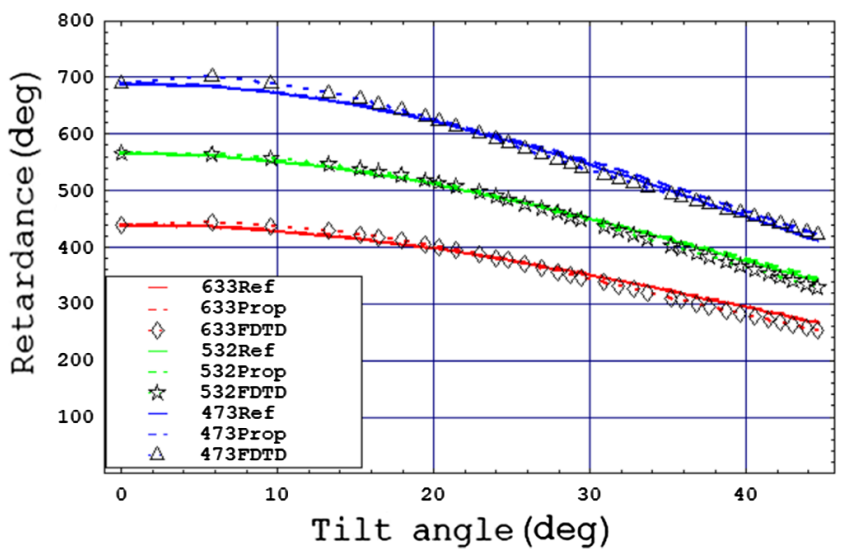

(b)

Fig. 9 SF-FDTD simulated experiment and prediction with the reference and proposed models for the wavelengths 633,532 , and $473 \mathrm{~nm}$ and for incidence at (a) 25 deg and (b) 45 deg.

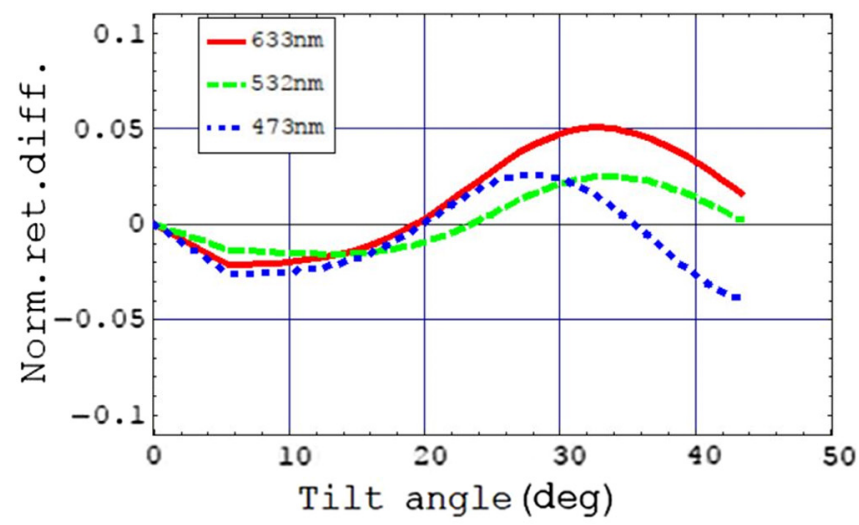

(a)

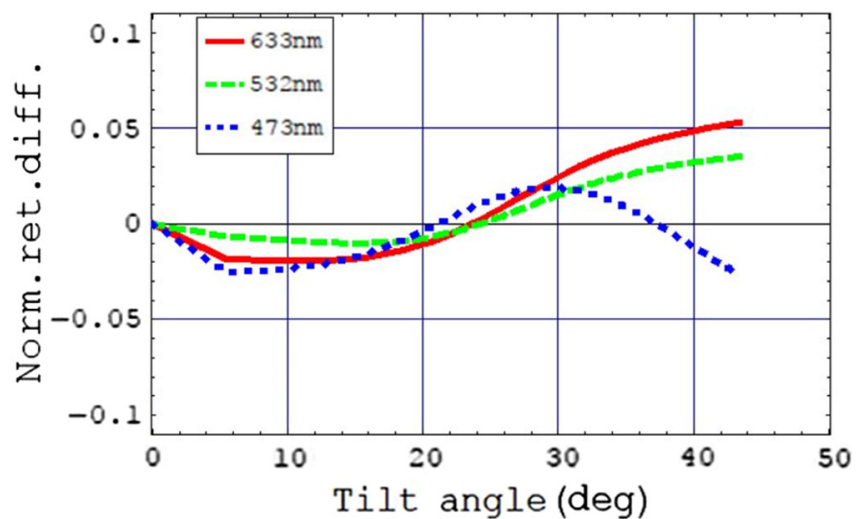

(b)

Fig. 10 For the reference model, difference between predicted and SF-FDTD experimental retardance normalized by the predicted value for wavelengths 633,532 , and $473 \mathrm{~nm}$ and for incidence at (a) $25 \mathrm{deg}$ and (b) 45 deg. 


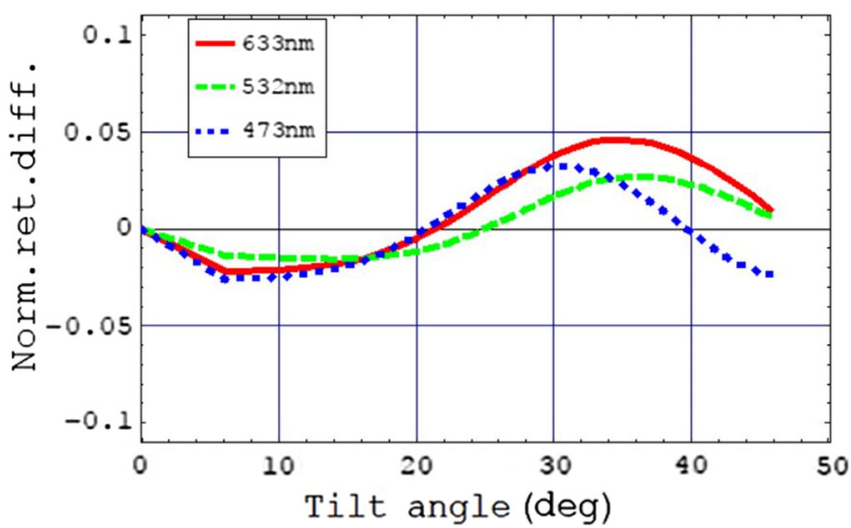

(a)

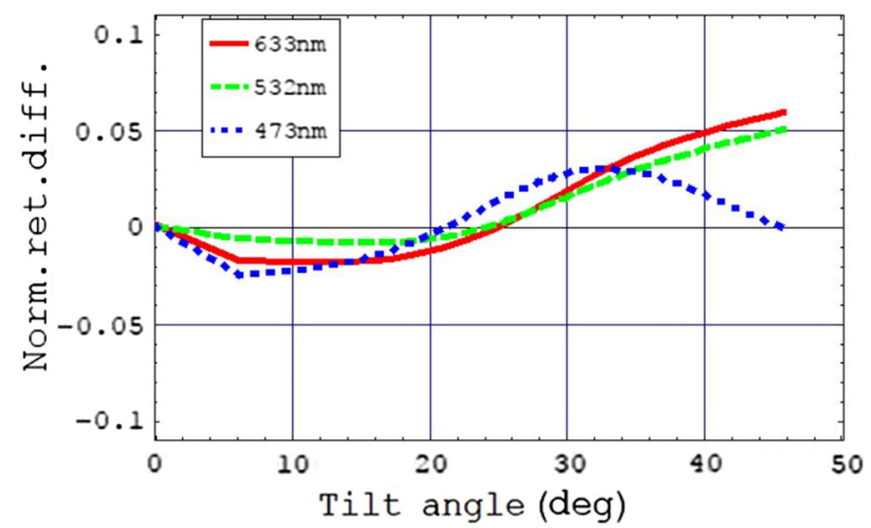

(b)

Fig. 11 For the proposed model, difference between predicted and SF-FDTD experimental retardance normalized by the predicted value for wavelengths 633,532 , and $473 \mathrm{~nm}$ and for incidence at (a) $25 \mathrm{deg}$ and (b) 45 deg.

In general, uncertainty increases with the increase in the tilt angle. These results agree with the ones we obtained previously from experimental retardance measurements. ${ }^{16}$

\section{Conclusions}

From the SF-FDTD-based computational evaluation that we have done in the present work, we clearly obtain that both simplified models are able to produce predictive retardance values; relative uncertainties are lower than $5 \%$ across the whole visible spectrum and for a wide range of incidence angles from $0 \mathrm{deg}$ to $45 \mathrm{deg}$. From the two models, the more simplified one that we propose is more robust since the values it provides for the parameters do not depend on the starting iteration values in the fitting procedure, i.e., it does not present ambiguities in the solutions. In the case of the reference model, for the off-state parameters, these ambiguities produce that in general the fitted values deviate from the true values. However, in the case of the proposed model, we have obtained that both the values obtained for OPD and for the tilt angle $\alpha$ coincide with the true values and are physically meaningful. These parameters are related to the birefringence, cell gap, and director profile. Therefore, the proposed model can be used as a means to inspect some important internal physical properties of the cell. We have also shown that the goodness in this calculation does not depend on the index of refraction considered for the LC, parameter $n_{\mathrm{LC}}$. This makes our proposed model easy and robust to apply, and a very useful option to characterize the possibilities of PA-LC devices in innovative applications, such as in experiments dealing with unconventional polarization states ${ }^{35,36}$ or in spectrum modulation. ${ }^{37}$

\section{Acknowledgments}

The work was supported by the Ministerio de Economía, Industria y Competitividad, Spain (Nos. FIS2014-56100C2-1-P and FIS2015-66570-P) and by the Generalitat Valenciana, Spain (No. PROMETEO II/2015/015).

\section{References}

1. G. Lazarev et al., "LCOS spatial light modulators: trends and applications," in Optical Imaging and Metrology: Advanced Technologies,
W. Osten and N. Reingand, Eds., pp. 1-29, Wiley-VCH Verlag GmbH \& Co. KGaA, Weinheim, Germany (2012).

2. A. Hussain et al., "Super resolution imaging achieved by using on-axis interferometry based on a spatial light modulator," Opt Express 21, 9615-9623 (2013)

3. K. Curtis et al., Eds., Holographic Data Storage: From Theory to Practical Systems, John Wiley \& Sons, Chichester, UK (2010).

4. F. J. Martínez et al., "Exploring binary and ternary modulations on a PALCoS device for holographic data storage in a PVA/AA photopolymer," Opt. Express 23, 20459-20479 (2015).

5. M. A. F. Roelens et al., "Dispersion trimming in a reconfigurable wavelength selective switch," J. Lightwave Technol. 26, 73-78 (2008).

6. M. Salsi et al., "Mode-division multiplexing of $2 \times 100 \mathrm{~Gb} / \mathrm{s}$ channels using an LCOS-based spatial modulator," J. Lightwave Technol. 30 , 618-623 (2012).

7. M. A. Solís-Prosser et al., "Preparing arbitrary pure states of spatial qudits with a single phase-only spatial light modulator," Opt. Lett. 38, 4762-4765 (2013).

8. N. Collings et al., "The applications and technology of phase-only liquid crystal on silicon devices," J. Display Technol. 7, 112-119 (2011).

9. Z. Zhang, Z. You, and D. Chu, "Fundamentals of phase-only liquid crystal on silicon (LCOS) devices," Light: Sci. Appl. 3, 1-10 (2014).

10. F. J. Martínez et al., "Retardance and flicker modeling and characterization of electro-optic linear retarders by averaged Stokes polarimetry," Opt. Lett. 39, 1011-1014 (2014).

11. A. Hermerschmidt et al., "Wave front generation using a phase-only modulating liquid-crystal based micro-display with HDTV resolution," Proc. SPIE 6584, 65840E (2007).

12. A. Lizana et al., "Influence of the temporal fluctuations phenomena on the ECB LCoS performance," Proc. SPIE 7442, 74420G (2009).

13. J. García-Márquez et al., "Flicker minimization in an LCoS spatial light Modulator," Opt. Express 20, 8431-8441 (2012).

14. K. Lu and B. E. A. Saleh, "Theory and design of the liquid crystal TV as an optical spatial phase modulator," Opt. Eng. 29, 240-246 (1990).

15. A. Márquez et al., "Quantitative prediction of the modulation behavior of twisted nematic liquid crystal displays based on a simple physical model," Opt. Eng. 40, 2558-2564 (2001).

16. F. J. Martínez et al., "Effective angular and wavelength modelling of parallel aligned liquid crystal devices," Opt. Lasers Eng. 74, 114-121 (2015).

17. P. Yeh and C. Gu, Optics of Liquid Crystal Displays, John Wiley \& Sons Inc., New York (1999).

18. A. Lien, "A detailed derivation of extended Jones matrix representation for twisted nematic liquid crystal displays," Liq. Cryst. 22, 171-175 (1997).

19. A. Márquez et al. "SF-FDTD analysis of a predictive physical model for parallel aligned liquid crystal devices," Proc. SPIE 10395, 1039509 (2017).

20. I Abdulhalim and D. Menashe, "Approximate analytic solutions for the director profile of homogeneously aligned nematic liquid crystals," Liq. Cryst. 37, 233-239 (2010).

21. A. Taflove and S. C. Hagness, Computational Electrodynamics: The Finite-Difference Time-Domain Method, 2nd ed., Artech House, Boston (2000).

22. C. Oh and M. J. Scuti, "Time-domain analysis of periodic anisotropic media at oblique incidence: an efficient FDTD implementation," Opt. Express 14, 11870-11884 (2006). 
23. J. Francés et al., "Acceleration of split-field finite difference timedomain method for anisotropic media by means of graphics processing unit computing," Opt. Eng. 53, 011005 (2014).

24. M. N. Miskiewicz, P. T. Bowen, and M. J. Escuti, "Efficient 3D FDTD analysis of arbitrary birefringent and dichroic media with obliquely incident sources," Proc. SPIE 8255, 82550W (2012).

25. J. Francés et al., "Split-field finite-difference time-domain method for second-harmonic generation in two-dimensionally periodic structures," J. Opt. Soc. Am. B 32, 664-669 (2015).

26. B. Xu et al., "Thin-slot formalism for the split-field FDTD analysis of periodic structure," Int. J. RF Microwave Comput. Aided Eng. 27, e21087 (2017).

27. J. Wang et al., "Efficient implementation of 2-D split-field FDTD method for analyzing periodic structures," IEEE Trans. Antennas Propag. 65, 3263-3266 (2017)

28. A. Lizana et al., "Influence of the incident angle in the performance of liquid crystal on silicon displays," Opt. Express 17, 8491-8505 (2009).

29. F. J. Martínez et al., "Averaged Stokes polarimetry applied to evaluate retardance and flicker in PA-LCoS devices," Opt. Express 22, 1506415074 (2014)

30. J. Li et al., "Refractive indices of liquid crystals for display applications," J. Disp. Technol. 1, 51-61 (2005).

31. F. Abeles, Optics of Thin Films in Advanced Optical Techniques, North-Holland Publishing Co., Amsterdam (1967).

32. W. H. Southwell, "Gradient-index antireflection coatings," Opt. Lett. 8, 584-586 (1983).

33. A. Márquez et al., "Analysis of Fabry-Perot interference effects on the modulation properties of liquid crystal displays," Opt. Commun. 265, 84-94 (2006).

34. J. L. Martínez et al., "Analysis of multiple internal reflections in a parallel aligned liquid crystal on silicon SLM," Opt. Express 22. 25866-25879 (2014).

35. T. G. Brown and Q. Zhan, "Focus issue: unconventional polarization states of light," Opt. Express 18, 10775-10776 (2010).

36. X. Zheng et al., "Compact LCOS-SLM based polarization pattern beam generator," J. Lightwave Technol. 33, 2047-2055 (2015).

37. I. Moreno et al., "Optical retarder system with programmable spectral retardance," Opt. Lett. 39, 5483-5486 (2014).

Andrés Márquez received his MSc and PhD degrees in physics from the Universidad Autónoma de Barcelona in 1997 and 2001, respectively. Since 2000, he has been with the Group of Holography and Optical Processing, Universidad de Alicante, where he is a professor of applied physics. His focus is in holographic recording materials, liquid crystal (LC) spatial light modulators (SLM), optical image processing and diffractive optics, with more than 100 articles in journals in the journal citation report (JCR), and more than 70 in proceedings of SPIE.

Jorge Francés received his PhD from the University of Alicante in 2011. He received his MSEE and BSEE degrees, both from the Technical University of Valencia, Valencia, Spain, in 2009 and 2006, respectively. Since 2008, he has been with the Group of Holography and Optical Processing, Universidad de Alicante, where he also works as a lecturer. His main research interests include physical optics, sound and vibration, and numerical simulation.
Francisco J. Martínez graduated in electronic engineering in 1996 and in physics in 1999 from the University of Valencia. He received his $\mathrm{PhD}$ from the University of Alicante in 2015. His research is focused on holography and holographic data storage systems. He has authored more than 10 scientific publications on the mentioned subjects in JCR indexed journals and more than 10 in Proceedings of SPIE. He is an assistant professor at the University of Alicante.

Sergi Gallego graduated in physics in 2001 from the University of Valencia, Spain, and received his PhD (with honors) from the University of Alicante, Spain, in 2005. His research is focused on holography, holographic recording materials, LCDs for holographic applications, electromagnetic theory, holographic and diffractive optical elements, and holographic data storage. He coauthored one patent and more than 80 scientific publications on the above-mentioned subjects in journals indexed in JCR (ISI).

Mariela L. Álvarez received her $\mathrm{PhD}$ in physics from the University of Alicante, Spain, in 2001. Since 2000, she has been a professor of applied physics at the University of Alicante. Her research is in the field of optics, with applications of scattering, holographic recording materials, holographic and diffractive optical elements, and holographic memories. She is coauthor of many scientific publications on the above-mentioned subjects in journals indexed in JCR (ISI)

Eva M. Calzado graduated in physics from the University of Valencia, Spain, in 2000 and received her $\mathrm{PhD}$ (with honors) from the University of Alicante, Spain, in 2008. Her research is focused on organic electronics, development of organic solid-state distributed feedback laser and material for laser applications identifying the presence of amplified spontaneous emission.

Inmaculada Pascual received her MSc and $\mathrm{PhD}$ degrees in physics from the University of Granada and University of Valencia in 1985 and 1990, respectively. In 1986, she joined the University of Alicante, where she was appointed as a full professor of optics in 2000. She has carried out research in holographic recording material, holographic memories, and optical elements in green photonics. This research has generated more than 125 publications in journals indexed in the JCR and 52 in Proceedings of SPIE.

Augusto Beléndez received his MSc and PhD degrees in physics from the University of Valencia in 1986 and 1990, respectively. In 1996, he became a full professor of applied physics at the University of Alicante. He is mainly interested in holography, holographic recording materials, holographic optical elements, LC SLMs, and diffractive optics. This research has generated more than 230 publications in journals indexed in the JCR and 75 in Proceedings of SPIE. 